\title{
EMISIÓN DE METANO ENTÉRICO EN GANADO BRAHMAN EN EL TRÓPICO DE COSTA RICA
}

\author{
Roberto Soto-Blanco ${ }^{1}$ y Sergio Abarca-Monge
}

\section{RESUMEN}

Emisión de metano entérico en ganado Brahman en el trópico de Costa Rica. En Costa Rica la producción bovina es la más difundida de las actividades agropecuarias, con la mayor área en uso agrícola bajo cobertura de pastizales (INEC 2014). Se realizaron dos experimentos para medir la emisión de metano de la fermentación entérica de bovinos cebú en la Estación Experimental Los Diamantes (EELD) del Instituto Nacional de Innovación y Transferencia en Tecnología Agropecuaria (INTA), entre noviembre de 2015 y febrero de 2017 en una zona de vida de Bosque Muy Húmedo Tropical Basal Holdridge (1978). El primero fue con vacas cebú adultas de la raza Brahman pastoreando Ratana (Ischaemum indicum); el segundo con machos Brahman en crecimiento en pasturas de Cayman híbrido (Hernández et al. 2014) en Pastoreo Racional Voisin con 42 días de rebrote. En el caso de los machos la carga animal durante la evaluación inició con 1,67 y finalizó con 2,47 UA ha-1 año-1. Los factores de conversión fueron $6,1 \%$ y 4,6\% de EB a metano y las emisiones en relación con el consumo de MS fueron de 20,0 y 14,5 $\mathrm{g} \mathrm{CH}_{4} / \mathrm{kg}$ MS para vacas en Ratana y machos en Cayman respectivamente.

Palabras clave: Factor Conversión Metano, Emisión Metano, Metano Brahman.

\section{INTRODUCCIÓN}

Los gases con efecto invernadero (GEI) se producen mediante procesos o mecanismos naturales, que han permitido a través del tiempo mantener una temperatura cálida en el planeta (González et al. 1999). Sin embargo, después de la revolución industrial se han evidenciado aumentos en la concentración de GEl en la atmósfera, situación que se asocia a efectos negativos en el medio ambiente, como aumento en la temperatura de la tierra y del océano, el descongelamiento de glaciares, aumento en el nivel del mar e incidencia de fenómenos naturales extremos como sequías y exceso de lluvia (Friedrinch-Wilhelm y Werner 2008; Shallcross et al. 2007).

Los GEl más relevantes son el dióxido de carbono $\left(\mathrm{CO}_{2}\right)$ el metano $\left(\mathrm{CH}_{4}\right)$ y el óxido nitroso $\left(\mathrm{N}_{2} \mathrm{O}\right)$, los cuales tienen un potencial de calentamiento de 1, 21 y 310, respectivamente (IPCC 2006). El metano además de que posee un mayor poder de calentamiento que el $\mathrm{CO}_{2}$, su tiempo de vida media en la atmósfera es de diez años (Moss et al, 2000;

1 Instituto Nacional de Innovación y Transferencia en Tecnología Agropecuaria, INTA. Costa Rica. rsoto@inta.go.cr, sabarca@inta.go.cr. 
Niggli et al. 2009). Dentro del sector agropecuario, el $\mathrm{CO}_{2}$ es generado en su mayoría por la combustión de combustibles fósiles (gasolina y diesel) utilizados en el equipo agrícola, el $\mathrm{CH}_{4}$ por la fermentación entérica que ocurre en el tracto digestivo de los rumiantes, y el $\mathrm{N}_{2} \mathrm{O}$ por el uso de fertilizantes nitrogenados en las actividades agrícolas (Cicerone y Oremland 1988). A nivel global se estima que las actividades agropecuarias producen el $25 \%$ de las emisiones de GEl, donde aproximadamente la mitad corresponden directamente a procesos pecuarios. Los bovinos son los animales que emiten la mayor emisión por su proceso de digestión (fermentación entérica), donde liberan metano, sumado a su cantidad y distribución geográfica global, dada la privilegiada habilidad de convertir pastos y forrajes en leche y carne (IPCC 2014).

Mediante la Convención Marco de las Naciones Unidas sobre Cambio Climático (CMNUCC), varios países han unido esfuerzos en busca de mitigar las emisiones de GEl de origen antropogénico. Un ejemplo es el tratado de Kioto celebrado en 1997, donde 196 países se comprometieron a realizar el inventario nacional de GEl y a reducir las emisiones en un 5\% con respecto a las emisiones de 1990 (Lascano y Cárdenas 2010). Costa Rica como país firmante de la convención, ha realizado tres inventarios nacionales utilizando la metodología propuesta por el Panel Intergubernamental de Expertos en Cambio Climático, (IPCC por sus siglas en inglés), el cual ha definido cuatro categorías como fuentes de emisión de GEl: energía; procesos industriales; agricultura, silvicultura y otros usos de la tierra (AFOLU) y desechos. De acuerdo con el inventario de 2010, la emisión de GEl de Costa Rica fue de 8788,84 Gg de $\mathrm{CO}_{2}$ equivalente, siendo el sector energía (combustibles y gases), desechos e industria, responsables del $80,6,15,7$ y $9,1 \%$ respectivamente. Mientras que el sector AFOLU aportó un valor negativo $(-473,29$ $\mathrm{Gg}$ ), debido a las grandes fijaciones de carbono que mantuvo (MINAE 2014). Dentro del total de metano emitido por AFOLU, la ganadería es responsable del $84 \%$, sin embargo, mediante el componente leñoso, fija el $48 \%$ del $\mathrm{CO}_{2}$ emitido por los otros sectores (INM 2014). Algunos investigadores coinciden que, en Costa Rica los sistemas de producción bovina y de rumiantes en general, son los que tienen el mayor potencial de reducir las emisiones de GEl (Abarca 2013).
En Costa Rica la producción bovina es la más difundida de las actividades agropecuarias, con la mayor área en uso agrícola bajo cobertura de pastizales (INEC 2014). Ésta aporta aproximadamente el 20\% de las emisiones del país, (2,3 millones de toneladas anuales de $\mathrm{CO}_{2}$ e) por sus procesos de producción (MINAE 2014). Por otra parte, se observa que el ganadero dedica tiempo a la protección de los bosques, la biodiversidad y el agua de sus fincas (Abarca 2014), siendo una actividad muy natural.

La variación en las emisiones de metano de los bovinos depende de la proporción de energía bruta contenida de los alimentos (EB) (RNC 2000) que utilicen los microorganismos del rumen en sus procesos metabólicos, incorporen en su biomasa y puedan liberar en forma de ácidos grasos, lo que se conoce como energía digestible (ED) (Ribeiro et al. 2015). Se estima que entre un 2 a un 12\% de la EB contenida en los alimentos se pierde como metano (Johnson y Johnson 1995).

Se han realizado pruebas para estimar las emisiones de metano de la fermentación entérica desde hace varios años (Hegarty 1999) principalmente con el fin de mejorar la nutrición de los animales, no obstante, la mayoría de las investigaciones han sido en latitudes diferentes a las tropicales, donde las razas de bovinos utilizadas no son comunes en el trópico y han sido alimentadas con granos y forrajes.

En algunos casos no se ha correlacionado la emisión con indicadores de producción durante los ensayos, ocasionando que los datos sean semejantes a los observados con dietas de otras latitudes e incluso a las estimadas por IPCC (2006), pero sin vinculación al crecimiento y producción de los animales. Estas investigaciones son interesantes entre pares de investigadores, pero generan muy poco aporte sustantivo a la realidad productiva, además pueden inducir a la toma de decisiones productivas erróneas cuando se está en un ambiente tropical.

Actualmente la ganadería bovina, tiene el reto de incrementar la productividad al menor costo posible y con la menor emisión de GEl (Montenegro y Abarca 2001). Favorablemente en los sistemas ganaderos del trópico húmedo, los animales interactúan con una gran cantidad de biomasa proporcionada por 
los árboles, arbustos, pastos y forrajes que existen en el ecosistema (Sorio 2012); que además de suministrar beneficios nutritivos a los animales, son capaces de retener gran cantidad de dióxido de carbono, siendo en algunos casos, mayor que la cantidad emitida por el mismo sistema de producción. Ante esta situación, se hace necesario cuantificar la retención y emisiones de $\mathrm{CO}_{2}$ equivalente en estos sistemas, con la finalidad de determinar su balance y en caso negativo, buscar alternativas tecnológicas y de manejo, que permitan reducir la emisión e incrementar la fijación de carbono.

El cuadro 1 presenta valores de emisión de metano de animales de razas cebuínas y algunos cruces alimentados con pastos tropicales y relación con la digestibilidad del forraje ofrecido, donde se observa una gran variabilidad entre investigaciones.

Cuadro 1. Emisión de metano obtenida por diferentes autores en ambientes y con pastos tropicales. Costa Rica. 2017.

\begin{tabular}{|c|c|c|c|c|c|c|}
\hline Tipo de animal & $\begin{array}{l}\text { Peso Vivo } \\
\text { (Kg) }\end{array}$ & Alimentación & $\begin{array}{l}\text { DIVMS } \\
(\%)\end{array}$ & $\begin{array}{c}\text { Emisión } \\
\text { (g CH4/a/d) }\end{array}$ & Localización & Referencia \\
\hline Holstein - Cebú & $373 \pm 37$ & Brachiaria no fertilizada & $41-50$ & 179 & Brasil & Pedreira et al 2009 \\
\hline Nelore & $402 \pm 52$ & $\begin{array}{l}\text { Heno de } B \text {. brizantha } \\
\text { rebrote } 15 \text { días }\end{array}$ & 64 & 133 & Brasil & Moysés 2007 \\
\hline Nelore & $402 \pm 53$ & $\begin{array}{l}\text { Heno de } B \text {. brizantha } \\
\text { rebrote } 45 \text { días }\end{array}$ & 63 & 138 & Brasil & Moysés 2007 \\
\hline Nelore & $402 \pm 54$ & $\begin{array}{l}\text { Heno de } B \text {. brizantha } \\
\text { rebrote } 90 \text { días }\end{array}$ & 63 & 134 & Brasil & Moysés 2007 \\
\hline Holstein - Cebú & 374 & B. decumbens sin fertilizar & & 190,5 & Brasil & Primavesi et al 2004 \\
\hline Cruce & 500 & B. brizantha & & 98 & África & Neto et al 2009 \\
\hline Cebú & $329 \pm 38$ & $\begin{array}{l}\text { Estabulado con heno } \\
\text { Digitaria decumbens }\end{array}$ & 63,3 & 181,5 & Costa Rica & INTA, 2015 \\
\hline Cebú & $329 \pm 39$ & $\begin{array}{l}\text { Estabulado con heno } \\
\text { Brachiaria arrecta }\end{array}$ & 47,4 & 110,4 & Costa Rica & INTA, 2015 \\
\hline Cebú & $329 \pm 40$ & Estabulado con heno Comercial & 47,5 & 125,8 & Costa Rica & INTA, 2015 \\
\hline
\end{tabular}

\section{MATERIALES Y MÉTODOS}

Las mediciones se llevaron a cabo en el área de ganadería de la Estación Experimental Los Diamantes (EELD), ubicada en Guápiles, cantón de Pococí, provincia de Limón; entre noviembre de 2015 y febrero de 2017. La zona de vida de acuerdo con la clasificación de Holdridge (1978) corresponde a Bosque Muy Húmedo Tropical Basal, con una precipitación anual de $4332 \mathrm{~mm}$ y una temperatura diaria promedio de $24,6^{\circ} \mathrm{C}$. Se realizaron dos experimentos en el mismo período: el primero fue con vacas cebú adultas multíparas de la raza Brahman pastoreando Ratana (Ischaemum indicum) con una carga animal de 2,0 UA/ha/año, con tres días de ocupación y 33 días de rebrote. El segundo grupo, con machos Brahman en crecimiento en pasturas de pasto Cayman híbrido de especies del género Brachiaria (Hernández et al. 2014) con tres años de establecido, en Pastoreo Racional Voisin (PRV) con 42 días de rebrote, la carga animal durante la evaluación inició con 1,67 y finalizó con 2,47 UA ha-1 año-1. En ambos casos la Unidad Animal (UA) fue definida en $450 \mathrm{~kg}$ de peso vivo y no se utilizó ningún tipo de fertilización durante el período de evaluación.

\section{Selección y manejo de los animales}

Se seleccionaron 16 animales; ocho hembras y ocho machos en crecimiento con un peso vivo promedio al inicio de las mediciones de 524,0 \pm 43,3 y $358,6 \pm 24,9 \mathrm{~kg}$ respectivamente, los cuales previamente recibieron adiestramiento para mejorar su docilidad y poner los collares recolectores de 
gases en sus cuellos con facilidad. Se tomaron muestras cada 33 días. El único alimento que consumieron los animales fue el forraje cosechado durante el pastoreo y la suplementación mineral ofrecida a libre voluntad. El acceso al agua fue sin restricción y los animales pastorearon con un buen confort y sin stress. Los animales seleccionados pastorearon con sus pares de hato, dentro del número y manejo normal de cada grupo.

\section{Medición de metano}

La medición de metano se realizó mediante la técnica del hexafloruro de azufre (SF6), la cual consistió en poner a nivel de rumen un tubo de permeación conteniendo SF6. La tasa de liberación del FS6 fue determinada por regresión lineal para cada tubo, los cuales se pesaron en forma semanal durante dos meses y medio previamente a ser implantados en el rumen de los animales. Para el muestreo se instaló un tubo colector al vacío y ergonómicamente diseñado en el cuello del animal, el cual fue conectado a un capilar que se situó por medio de un gamarrón (bozal) en el morro del animal (Westberg et al. 1998 y MPI 2014). Esta técnica permitió el pastoreo libre y sin limitaciones de los animales, garantizando un comportamiento y bienestar adecuado y natural. Se utilizó un tubo colector de gases por día de pastoreo. El metano y FS6 contenidos en el tubo colector se determinaron por cromatografía de gases en el laboratorio del INTA de Costa Rica. Se asumió que las tasas de emisión de FS6 y $\mathrm{CH} 4$ eran exactamente iguales, entonces las diluciones también fueron idénticas. La mezcla de los gases se debió a los movimientos de los animales y del mismo rumen. Por lo tanto, la tasa de emisión del metano $\left(Q_{\mathrm{CH}}\right)$ puedo ser calculada por la medición de la concentración de $\mathrm{CH} 4$ y FS6, así como la tasa de liberación de FS6 (Q sF6):

$Q \mathrm{CH} 4=\mathrm{Q}$ SF6 $\times$ [CH4] / [SF6] (Westberg et al. 1998).

\section{Degradación "in situ" y calidad nutritiva}

Para la determinación de la degradación "in situ" de los forrajes que consumieron los animales a los que se les medió la emisión de $\mathrm{CH}_{4}$ por medio de la técnica del FS6, se utilizaron cuatro novillos con cánula ruminal, con un peso aproximado de $350 \mathrm{~kg}$, los cuales se mantuvieron pastoreando las mismas pasturas que los animales de medición directa de $\mathrm{CH}_{4}$, así como en las mismas condiciones agroecológicas y de manejo. El período de incubación fue de 48 horas, con material molido a $2 \mathrm{~mm}$ ( $5 \mathrm{~g}$ de forraje) en bolsas de nylon de aproximadamente $17,0 \mathrm{~cm}$ de largo por 9,0 cm de ancho, con un poro de $52 \mu$, de acuerdo con la técnica descrita por Orskov et al (1980). El cálculo de degradación para la materia seca (MS) se realizó mediante la siguiente fórmula:

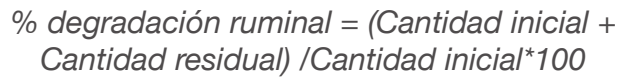

\section{Estimación del consumo voluntario de materia seca}

El consumo voluntario se estimó mediante la técnica de marcadores externos utilizando óxido crómico $\left(\mathrm{Cr}_{2} \mathrm{O}_{3}\right)$ como marcador (Mejía 2002). A los animales se les suministró una dosis de $10 \mathrm{~g} \mathrm{~d}^{-1}$ de $\mathrm{Cr}_{2} \mathrm{O}_{3}$ durante 7 días consecutivos, tomando muestras de heces directamente del recto del animal a partir del quinto día. A las muestras de forraje y heces se les determinó carbono (C) y nitrógeno (N) mediante un análisis realizado en el laboratorio ubicado en el CATIE. 


\section{RESULTADOS Y DISCUSIÓN}

\section{Vacas con manejo tradicional en pasturas de Ratana (Ischaemum indicum)}

La calidad nutritiva de la pastura se presenta en el cuadro 2. Los contenidos de Proteína Cruda (PC) y Fibra Detergente Neutro (FDN), son concordantes con los observados por Villalobos (2010) para este tipo de pastura en condiciones de trópico muy húmedo y edad de rebrote similar.

Cuadro 2. Calidad nutritiva de la pastura de Ratana (Ischaemum indicum). Guápiles, Costa Rica. 2017.

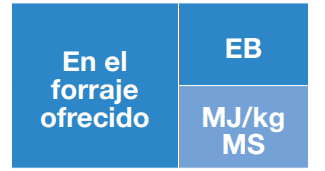

\begin{tabular}{lc} 
Promedio & 18,2 \\
$\begin{array}{l}\text { Desviación } \\
\text { estándar }\end{array}$ & 0,3 \\
\hline
\end{tabular}

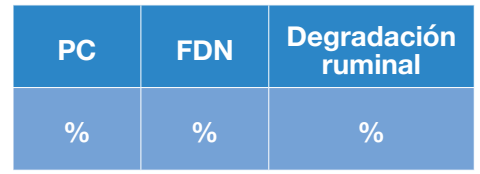

\begin{tabular}{rrr}
13 & 56,7 & 54,3 \\
1,5 & 3,2 & 12,2 \\
\hline
\end{tabular}

Durante el período experimental las vacas mantuvieron una condición corporal, estado sanitario y reproductivo muy bueno pese a su edad (7 - 9 años). En relación con la emisión de $\mathrm{CH}_{4}$, se observó diferencias significativas entre períodos de medición pero no entre vacas. El promedio de emisión fue de 204,5 g CH 4 vaca $^{-1}$ día $^{1}$, siendo ligeramente mayor a la observada por INTA (2015); Pedreira et al. (2009) y Primavesi et al. (2004) con animales de pesos inferiores (entre 329 y $374 \mathrm{~kg}$ ) a los utilizados en esta evaluación, en el primer caso con una digestibilidad de forraje ofrecido mucho mayor (63,3 \%) y en el último con una semejante (50 \%) al valor determinado en la pastura utilizada en esta investigación. Al relacionar la emisión por unidad de peso vivo, se observó que bajo las condiciones del presente estudio, se obtuvo una relación más baja $\left(0,39 \mathrm{~g} \mathrm{CH}_{4} \mathrm{~kg} \mathrm{PV}^{-1}\right)$ que las obtenidas por INTA (2015), Pedreira et al. 2009 y Primavesi et al. (2004) pero similar a la obtenida por Moysés (2007) con animales cebú de la raza Nelore.
Cuadro 3. Emisión de metano de vacas en pastura de Ratana (Ischaemum indicum) Guápiles, Costa Rica. 2017.

\begin{tabular}{l|c|c|c}
\hline \multicolumn{1}{c|}{ Variable } & Unidades & Promedio & $\begin{array}{c}\text { Desviación } \\
\text { Destándar }\end{array}$ \\
\hline $\begin{array}{l}\text { Consumo de } \\
\text { materia seca }\end{array}$ & Kg/animal/día & 11,8 & 1,0 \\
\cline { 2 - 4 } & \%Peso Vivo & 2,2 & 0,1 \\
\hline $\begin{array}{l}\text { Emisión de } \\
\text { metano }\end{array}$ & g/vaca/día & 204,5 & 148,3 \\
\cline { 2 - 4 } $\begin{array}{l}\text { Energía bruta } \\
\text { convertida } \\
\text { a metano }\end{array}$ & \% & 20,0 & 12,0 \\
\hline
\end{tabular}

En hembras adultas de las razas cebú, el comportamiento por la jerarquía del hato y el estado fisiológico del animal (celo, parto, amamantamiento) juegan un papel importante en el consumo voluntario y la calidad de la MS a las que tienen acceso, lo que genera una mayor variación entre y dentro de animales en igualdad de disponibilidad forrajera, lo que explicaría observar variaciones de emisión para una misma vaca durante el período de evaluación. Lo anterior sumado a la variación en la oferta de forraje, de acuerdo con la época del año y las condiciones de lluvia intensa en la zona.

\section{Machos en crecimiento en pasturas de Cayman (Brachiaria híbrido)}

La disponibilidad de forraje en MS, su degradación ruminal a 48 horas, así como los contenidos de PC y FDN se presentan el cuadro 4. Estos son concordantes con los obtenidos por Hernández et al. (2014) para la misma pastura. 
Cuadro 4. Disponibilidad de materia seca y calidad nutritiva de Cayman. Guápiles, Costa Rica. 2017.

\begin{tabular}{|c|c|c|c|c|c|}
\hline \multirow{2}{*}{$\begin{array}{l}\text { Forraje } \\
\text { ofrecido }\end{array}$} & MS & EB & PC & FDN & $\begin{array}{l}\text { Degradación } \\
\text { ruminal }\end{array}$ \\
\hline & $\begin{array}{l}\text { kg } \\
\mathrm{MS} \\
\mathrm{ha}^{-1}\end{array}$ & $\begin{array}{l}\text { MJ } \\
\mathbf{k g} \\
\mathbf{M S}^{-1}\end{array}$ & \multicolumn{3}{|c|}{$\%$} \\
\hline Promedio & 6211 & 18,0 & 9,6 & 56,6 & 59,7 \\
\hline $\begin{array}{l}\text { Desviación } \\
\text { estándar }\end{array}$ & 2612 & 0,4 & 1,9 & 5,3 & 4,3 \\
\hline
\end{tabular}

El consumo promedio estimado de MS fue de 12,8 $\mathrm{kg} \mathrm{MS} \mathrm{a}^{-1} \mathrm{~d}^{-1}$, siendo equivalente a 24,0 $\mathrm{g}$ $\mathrm{kg}^{-1}$ peso vivo $(2,4 \% \mathrm{PV})$ semejante al reportado por Detmann et al. (2014) de 24,8 y $21,8 \mathrm{~g} / \mathrm{kg}$ en general y animales en crecimiento respectivamente. Los consumos de MS observados se asociaron a una alta disponibilidad forrajera $(3,5-4,7 \mathrm{t} / \mathrm{ha} /$ pastoreo) durante todo el período de evaluación, al relacionar esta alta oferta de forraje con su calidad, se podría presumir que el potencial de selección de los animales pudo influir en una mejora de la calidad del forraje consumido, especialmente en los contenidos de PC y valores de degradación ruminal.
El factor de conversión de EB consumida a energía liberada en forma de $\mathrm{CH}_{4}$ en vacas de la raza Brahman en condiciones de trópico muy húmedo y consumiendo pasto Ratana (I. indicum) fue de $6,1 \%$, siendo menor al $6,5 \%$ que establece IPCC (2006) como promedio para animales en pastoreo. Por otro lado, el factor de conversión de EB consumida a energía liberada en $\mathrm{CH}_{4}$ para machos en crecimiento de la raza Brahman en condiciones de trópico muy húmedo consumiendo pasto Cayman (Brachiaria híbrido) fue de 4,6\%, siendo 29,2\% menor al que establece IPCC (2006) como promedio para animales en pastoreo e incluso más bajo del 5,5\% que establece como límite inferior.

La pastura de pasto Cayman independientemente del manejo, suministró mejor calidad y cantidad de alimento que la de Ratana.

La relación entre el nitrógeno consumido y el excretado concuerda con lo reportado por Moran y Vercoe (2009), que sugieren que el ganado cebú podría tener una mayor digestibilidad verdadera del nitrógeno y un menor metabolismo fecal que las razas originarias de Europa. Así mismo, la proporción de nitrógeno excretado en el presente estudio concuerda con lo reportado por Kennedy (2013). En relación con los valores de carbono excretados, estos concuerdan con los reportados por Pelster et al. (2016) en pasturas tropicales del este de África.

Cuadro 5. Estimación de materia seca carbono y nitrógeno, consumidos y excretados. Guápiles, Costa Rica. 2017.

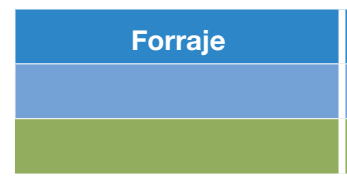

Promedio

Desviación estándar

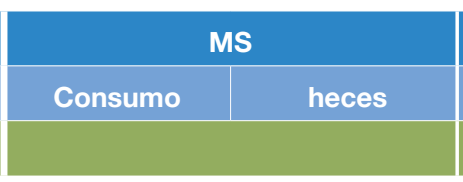

12,8

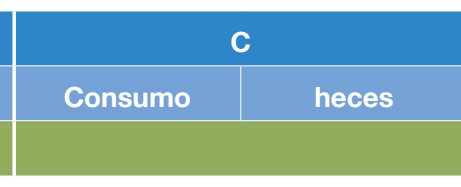

5,7

1,0

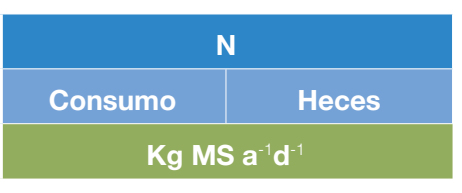

0,17

0,03
0,09 0,02
De acuerdo con Van Soest (1994) el consumo de forrajes esta inversamente relacionado con el tiempo de retención y la degradación de la MS en el rumen. Por otro lado, se encontraron diferencias en relación con el consumo y tasa de pasaje con animales de diferentes razas, donde la raza Bon (criollo colombiano) obtuvo como norma en varias localidades y calidad de pasturas tropicales un mayor consumo y una menor velocidad de pasaje (Giraldo et al 2013).
De acuerdo con Oliveira et al. (1980), comparando animales Bos taurus y Bos indicus, alimentados con pasto Napier (Pennisetum purpureum) obtuvo un mayor consumo y producción de heces en animales $B$. taurus, con respecto a los $B$. indicus, no obstante, estos últimos presentaron mayor variación diaria. 
El contenido de EB del forraje fue inferior en un 6,6\% al sugerido por NRC (2001) de 4,4 Mcal/kg MS y semejante a los valores reportados por Cardona et al. (2002) para pasturas tropicales en Colombia y Cota et al. (2014) con Brachiaria brizantha en Brasil.

Cuadro 6. Emisión de gramos de metano en machos pastoreando Cayman (Brachiaria híbrido) Guápiles, Costa Rica. 2017.

\begin{tabular}{l|c|c|c}
\hline \multirow{2}{*}{ Variable } & Unidades & Promedio & $\begin{array}{c}\text { Desviación } \\
\text { estándar }\end{array}$ \\
\hline $\begin{array}{l}\text { Consumo de } \\
\text { materia seca }\end{array}$ & Kg/animal/día & 12,8 & 2,4 \\
\cline { 2 - 4 } & $\% \mathrm{PV}$ & 2,6 & 0,2 \\
\hline $\begin{array}{l}\text { Emisión de } \\
\text { metano }\end{array}$ & g/animal/día & 168,5 & 127,7 \\
\hline $\begin{array}{l}\text { Energía bruta } \\
\text { convertida } \\
\text { a metano }\end{array}$ & g/kg MS & 14,5 & 9,4 \\
\hline
\end{tabular}

Los contenidos de EB de los forrajes fueron menores a los indicados en la literatura convencional, basada en dietas con granos y forrajes de otras latitudes.

Aún cuando las emisiones en promedio no fueron elevadas en ninguna etapa de crecimiento de los animales, la conversión de $\mathrm{EB}$ a $\mathrm{CH}_{4}$ fue mayor en etapas tempranas y no fue lineal durante el período de evaluación. La evaluación se realizó en las condiciones más naturales posibles, lo que se refleja en las buenas ganancias de peso obtenidas en los animales en crecimiento alimentados con pasto Cayman y el buen estado de las vacas que consumieron pasto Ratana.

En general, el promedio de emisión diaria por animal para el período estudiado fue bajo. No se observó tendencia o relación significativa entre la emisión y el peso vivo durante el período de crecimiento de los animales. El promedio general para emisión en relación con el consumo de alimento se consideró adecuado.
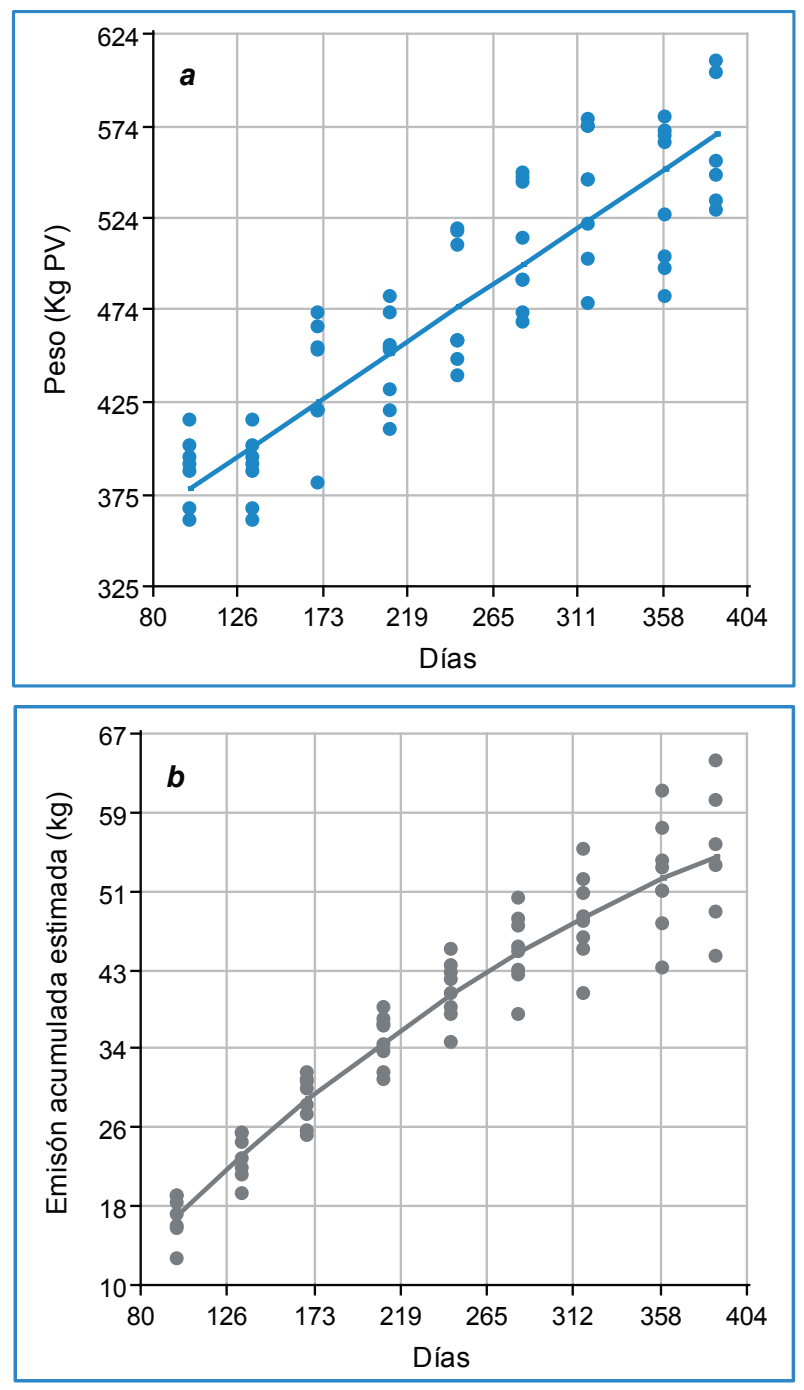

Incremento de PV (a) y emisión de $\mathrm{CH}_{4}$ acumulada

(b) en función de los días de PRV en pasto

Cayman. Guápiles, Costa Rica. 2017.

Durante el período de estudio, se observó una tendencia lineal significativa $(p<0001)$ para la ganancia de peso, la cual mantuvo una tasa de incremento de 0,670 kg/día, mientras la emisión de $\mathrm{CH}_{4}$ acumulada mantuvo una tendencia también significativa incremental pero cuadrática, lo que sugiere que a medida que los animales crecieron y alcanzaron mayor peso y edad, incrementaron la eficiencia de conversión de la energía del pasto. No obstante, es posible que en edades tempranas cercanas al destete, la eficiencia de conversión del pasto sea relativamente baja, produciendo mayor cantidad de $\mathrm{CH}_{4}$ en relación con el consumo de MS y al PV, sugiriendo la posibilidad de que a estas edades iniciales, los animales Brahman no aprovechan eficientemente la energía suplida por el pasto en condiciones tropicales. 


\section{LITERATURA CITADA}

Abarca, S. 2013. Cambio climático y mitigación en fincas lecheras. Revista Universidad Técnica Nacional 15(63):28-31.

Abarca, S. 2014. Servicios eco sistémicos en fincas ganaderas. Biodiversidad: Fauna (en línea). Horizonte Lechero. 3 ed. Ciudad, país. Paginación. Consultado 20 mar. 2017. Disponible en https://issuu.com/ proleche/docs/revista_horizonte_diciembre_2014

Cardona, M; Sorza, J; Posada, S; Carmona, J; Ayala, S; Alvarez, O. 2002. Establecimiento de una base de datos para la elaboración de tablas de contenido nutricional de alimentos para animales (en línea). Revista Colombiana de Ciencias Pecuarias 15(2):240246. Consultado 20 mar. 2017. Disponible en https:// dialnet.unirioja.es/servlet/articulo?codigo=3242962

Cicerone, R; Oremland, R. 1988. Biogeochemical aspects of atmospheric ethane. Global Biogeochem (en línea). Cycles 2:299-327. Consultado 20 mar. 2017. Disponible en http://web.altagenetics.com/ mexico/DairyBasics/Details/9486_Ganaderia-bovinay-emision-de-gases-de-efecto-invernadero.html

Cota, O; Figueiredo, D; Branco, R; Magnani, E; Ferreira, C; Freitas, L; Zerlotti, M. 2014. Methane emission by Nellore cattle subjected to different nutritional plans (en línea). Tropical Animal Health and Production 46(7):1229-1234. Consultado 20 mar. 2017. Disponible en https://link.springer.com/ article/10.1007/s11250-014-0632-3

Detmann, E; Valente, E; Batista, E; Huhtanen. 2014. An evaluation of the performance and efficiency of nitrogen utilization in cattle fed tropical grass pastures with supplementation (en línea). Livestock Science 162:141-153. Consultado 20 mar. 2017. Disponible en http://www.livestockscience.com/article/S1871 -1413(14)00083-3/pdf

Friedrich-Wilhelm, G; Werner, P. 2008. Climate development in the last century-global regional. International Journal of Medical Microbiology 298:5-11.

González, BF; Rodríguez, H. 1999. Proyección de las emisiones de gases de efecto invernadero (GEI), Colombia 1998-2010. Revista Académica Colombiana de Ciencias 23(89):497-505.

Giraldo, L; Carulla, J; Calle, M. 2013. Metabolismo digestivo de razas bovinas (bon, Holstein, cebú) en pasturas tropicales de calidad contrastante. In: Consideraciones sobre el mejoramiento genético y factores asociados en bovinos criollos colombianos y grupos multirraciales. Campos, R; Durán, CV (eds.). Bogotá, Colombia. p.103-127.

Hegarty, R. 1999. Mechanisms for competitively reducing ruminal methanogenesis. Australian Journal of Agricultural Research. 50(8):1299-1305.

Hernández, M; Abarca, S; Soto, R. 2014. Evaluación de pasto Brachiaria híbrido cv Cayman, en pastoreo en el trópico muy húmedo de Costa Rica. Revista Universidad Técnica Nacional 16(70):48-54.

Holdridge, L 1978. Ecología basada en zonas de vida. San José, Costa Rica. 216 p.

INEC (Instituto Nacional de Estadística y Censos, CR). 2014. VI Censo Nacional Agropecuario (en línea). San José, Costa Rica. Consultado 19 may. 2016. Disponible en http://inec.cr/censos/ censo-agropecuario-2014

INTA (Instituto Nacional de Innovación y Transferencia en Tecnología Agropecuaria). 2015. Determinación de la emisión de metano en novillos Brahman en el ecosistema de bosque seco tropical de Costa Rica Montenegro, J; Barrantes, E (eds.). (Informe). INTA UGIT. 23p.

IPCC (Panel Intergubernamental de Cambio Climático), 2006. Directrices para los inventarios nacionales de gases de efecto invernadero (en línea). In Agricultura, silvicultura y otros usos de la tierra. Emisiones resultantes de la gestión del ganado y del estiércol (Cap.10). Hayama, Japón. 10.31-10.34. Consultado 20 mar. 2017. Disponible en http://www. ipcc-nggip.iges.or.jp/public/2006gl/index.html

IPCC (Panel Intergubernamental de Cambio Climático), 2014. Cambio climático 2014: Informe de síntesis. (en línea). In Pachauri, RK; Meyer, LA (eds.). Contribución de los Grupos de trabajo I, II y III al Quinto Informe de Evaluación del Grupo Intergubernamental de Expertos sobre el Cambio Climático. Ginebra, Suiza, 157 p. Consultado 20 mar. 2017. Disponible en https://www.ipcc.ch/pdf/assessment-report/ar5/ syr/SYR_AR5_FINAL_full_es.pdf

Johnson, K., Johnson, D. 1995. Methane emissions from cattle. Journal of Animal Science 73:2483-2492.

Kennedy, WK. 2013. Nitrogen Metabolism in Bos indicus and Bos taurus cattle consuming low - quality forage. Tesis Mag. Sc. Texas, Estados Unidos de América.Texas A\&M University. 76 p. 
Lascano, CE; Cárdenas, E. 2010. Alternatives for methane emissions mitigation in livestock systems. Revista Brasilera de Zootecnia 39:175-182.

Mejía, HJ. 2002. Consumo voluntario de forrajes en pastoreo. In Acta Universitaria 12(3):56-65.Irapuato México. Universidad de Guanajuato. Instituto de Ciencias Agrícolas.

MINAE (Ministerio de Ambiente y Energía). 2014. Inventario nacional de gases de efecto invernadero y absorción de carbono 2010. San José, Costa Rica. $64 \mathrm{p}$.

Montenegro, J; Abarca S. 2001. Importancia del sector agropecuario costarricense en la mitigación del calentamiento global. Ministerio de Agricultura y Ganadería, Instituto Meteorológico Nacional. San José, Costa Rica. 96 p.

Moran, JB; Vercoe, J.E. (2009). Some factors affecting apparent nitrogen digestibility of roughage diets fed to cattle. The Journal of Agricultural Science 78(2):173-177.

Moss, A; Jouany, J; Newbold, J. 2000. Methane production by ruminants: its contribution to global warming. Annimal. Zootechnique 49:231-253.

MPI (Ministry for Primary Industries New Zealand). 2014. Guidelines for use of sulphur hexafluoride (FS6) tracer technique to measure enteric methane emissions from ruminants.166p. Consultado 16 mar. 2018. Disponible en https://globalresearchalliance. org/wp-content/uploads/2012/03/SF6-Guidelines-allchapters-web.pdf

Moysés, C. 2007. Emissão de metano por bovinos Nelore ingerindo Brachiaria brizantha em diferentes estádios de maturação. Tesis Mag. Sc. Sao Paulo, Brasil. Faculdade de Medicina Veterinária e Zootecnia da Universidade de São Paulo. 67 p.

Niggli, U; Fliebbach, A; Hepperly, P; Scialabba, N. 2009. Low Greenhouse gas Agriculture: Mitigation and adaptation potential of sustainable farming systems. FAO Review (2009) Roma (Italia). Organización de las Naciones Unidas para la Agricultura y la Alimentación (FAO) (en línea). Consultado 12 jun. 2018. Disponible en http://www.fao.org/tempref/docrep/fao/010/ai7 81e/ai781e00.pdf

NRC (National Research Council), 2001. Nutrient Requirements of Dairy Cattle. Ciudad, Estados Unidos de Norte América.353 p.

Oliveira, M; Veiga, J; Rocha, U. 1980. Estudo comparativo da ingestão de alimentos eliminacao fecal em
Bos taurus e Bos indicus. Revista Facultada de Veterinaria, Universidad Sao Pablo 17: 19-27.

Orskov, ER; Hovell, RO; Mould, F. 1980. Uso de la técnica de la bosa de nylon para la evaluación de los alimentos. Producción Animal Tropical 5(3):213-233.

Pedreira, M; Primavesi, O; Aparecida Lima, M; Frighetto, R; de Oliveira, S; Berchielli, T. 2009. Ruminal methane emission by dairy cattle in Southeast Brazil. Scientist Agriculture 66(6):742-750.

Pelster, D; Gisore, B; Koske, J; Goody, J; Korir, D; Rufino, D; Butterbach-Bahl, K. 2016. Methane and nitrous oxide emission from cattle excreta on an East African grassland. Journal Environmental Quality 45(5):1531-1539.

Primavesi, O; Shiraishi, R; Pedreira, M; de Lima, M; Berchielli, T; Barbosa, P. 2004. Metano entérico de bovinos leiteiros em condições trapicáis brasileiras. Pesquisa Agropecuaria Brasileira 39(3):277-283.

Ribeiro PLG; Machado FS; Campos MM; Guimaraes RG; Tomich TR; Larissa G Reis LG; Pharm; Cassius C. 2015. Enteric methane mitigation strategies in ruminants: a review. Revista Colombiana de Ciencias Pecuarias 28:124-143.

RNC (National Research Council), 2000. Agriculture Nutrient. Subcommittee on Beef Cattle Nutrition Committee on Animal Nutrition Board on Requirements of Beef Cattle. $7^{\text {th }}$ Ed. National Academies Press, Washington, D.C, Estados Unidos de Norte América. 232 p.

Shallcross, D; Khalil, M; Butenhoff, C. 2007. The atmospheric methane sink. In Greenhouse Gas Sink. Reay, DS; Hewitt, CN; Smith, KA; Grace, J. (eds.). Wallingford, UK. CAB International.

Sorio, H. (2012). Pastoreo Voisin. Teorías-PrácticasVivencias (3 ed.). Passo Fundo: Meritos. Brasil. 298 p.

Van Soest, P. 1994. Nutritional Ecology of the Ruminant. 2 ed. Ithaca. New York Comstock Cornell University Press. 476p.

Villalobos, E. 2010. Respuesta del pasto Ratana (Ischaemum indicum) a la fertilización nitrogenada y edad de rebrote. Tesis Ing. Agr. San Carlos, Costa Rica. Instituto Tecnológico de Costa Rica. 45 p.

Westberg, $\mathrm{HH}$; Johnson, KA; Cossalman, MW; Michal, JJ. 1998. A SF6 tracer technique: methane measurement from ruminants. Washington State University. Pullman, Washington, Estados Unidos de América. $40 \mathrm{p}$. 
